\title{
Mosquito salivary gland protein preservation in the field for immunological and biochemical analysis
}

\author{
A Fontaine, A Pascual, I Diouf, N Bakkali, S Bourdon, T Fusai, C Rogier, L Almeras*
}

\begin{abstract}
Mosquito salivary proteins are involved in several biological processes that facilitate their blood feeding and have also been reported to elicit an IgG response in vertebrates. A growing number of studies have focused on this immunological response for its potential use as a biological marker of exposure to arthropod bites. As mosquito saliva collection is extremely laborious and inefficient, most research groups prefer to work on mosquito salivary glands (SGs). Thus, SG protein integrity is a critical factor in obtaining meaningful data from immunological and biochemical analysis. Current methodologies rely on an immediate freezing of SGs after their collection. However, the maintenance of samples in a frozen environment can be hard to achieve in field conditions. In this study, SG proteins from two mosquito species (Aedes aegypti and Anopheles gambiae s.s.) stored in different media for 5 days at either $+4^{\circ} \mathrm{C}$ or room temperature (RT) were evaluated at the quantitative (i.e., ELISA) and qualitative (i.e., SDSPAGE and immunoblotting) levels. Our results indicated that PBS medium supplemented with an anti-protease cocktail seems to be the best buffer to preserve SG antigens for 5 days at $+4^{\circ} \mathrm{C}$ for ELISA analysis. Conversely, celllysis buffer (Urea-Thiourea-CHAPS-Tris) was best at preventing protein degradation both at $+4^{\circ} \mathrm{C}$ and RT for further qualitative analysis. These convenient storage methods provide an alternative to freezing and are expected to be applicable to other biological samples collected in the field.
\end{abstract}

\section{Findings}

Mosquitoes are responsible for a wide range of important diseases that cause morbidity and mortality in tropical and temperate regions [1,2]. Pathogen transmission occurs during the blood-feeding of infected mosquitoes, concomitant with salivary protein release [3]. Analysis of salivary mosquito contents using transcriptomic and proteomic tools [4-6] have revealed a panel of salivary molecules with anti-hemostatic and immuno-modulatory properties which facilitate blood meals by counteracting host's defences [6,7]. It was repeatedly demonstrated that mosquito salivary proteins could also elicit a host IgG response in natural conditions [8-10]. Thus, the potential use of these antigenic proteins as epidemiological markers for evaluating individual human exposure level to specific mosquito species is a major research area. Additionally, the identification of

\footnotetext{
* Correspondence: almeras|@imtssa.fr

Unité de recherche en biologie et épidémiologie parasitaires (URBEP) -

UMR6236 - IFR48, Antenne Marseille de I'Institut de Recherche Biomédicale des Armées (IRBA), Le Pharo, BP 60109, 13262 Marseille Cedex 07, France
}

such vector-borne immunogenic proteins can lead to a panel of promising applications such as the evaluation of anti-mosquito strategies effectiveness, the mapping of new infestation areas, the estimation of disease transmission risk or the development of vaccines protecting the host against the transmission and establishment of pathogens $[11,12]$. As our aim is to identify biological markers of individual exposure to arthropod bites using correspondent antigenic materials, it was necessary to develop a convenient protocol to collect and preserve biological samples in the field.

The most common method used to obtain salivary proteins is salivary gland (SG) dissection [13-15]. Mosquito SGs contain a cocktail of enzymes and active proteins necessary for their blood-feeding that could alter salivary protein integrity $[3,16]$. To avoid protein degradation, SGs are generally collected on ice and stored at or below $-20^{\circ} \mathrm{C}$ until needed $[15,17]$. However, maintaining samples in a frozen environment can be hard to achieve in field conditions.

\section{C) Biomed Central}


Although hundreds of mosquito species have been reared in laboratories, relatively few have been continuously maintained through several generations in a caged environment [18]. Furthermore, continuous mass rearing is a tremendous task that requires particular skills and time and is also subject to biosafety considerations. Thus, to avoid the risk of mosquito settlement by bringing larvae outside their natural habitat in an area exempt of this species, collection of SG in the field appears more reasonable. However, SG dissection currently necessitates a large number of living mosquitoes in situ in close proximity to a freezer system to prevent protein degradation. Yet, in inter-tropical areas, mainly in sub-Saharan regions, some villages are highly isolated and frozen apparatus are not always available. Additionally, transport of SGE until a freezer system could take several hours and a continuous sample freezing could be uncertain. Therefore, alternative and convenient procedures need to be developed to preserve biological materials when a continuously cold environment would be hard to maintain. To this end, the preservation of SG proteins from two mosquito species (Anopheles gambiae s.s and Aedes aegypti) in different storage mediums and temperature conditions over 5 days have been evaluated quantitatively (i.e., ELISA) and qualitatively (i.e., SDSPAGE and immunoblotting).

SGs from non-blood fed, 5-8 day-old adult An. gambiae s.s. (Kisumu strain [19]) and Ae. aegypti (Bora-Bora strain [20]) female mosquitoes bred in a laboratory under standard conditions (i.e., $26^{\circ} \mathrm{C}$ and $60 \%$ humidity) at the "Institut de Recherche pour le Développement" (IRD, Montpellier)were dissected under a stereomicroscope, as previously described [15]. For each species, a total of 30 pairs of SGs were pooled and stored in each buffer and temperature condition. Briefly, samples were collected and stored 5 days at $+4^{\circ} \mathrm{C}$ or RT (about $21^{\circ} \mathrm{C}$ ) either in a Phosphate Buffered Saline buffer supplemented with an anti-protease cocktail (one tablet of Complete, EDTA-free protease inhibitor cocktail (Roche Diagnostics, Indianapolis, USA) in $5 \mathrm{ml}$ of PBS, PBSpi buffer), or in a cell lysis buffer (8 M urea (Sigma), $2 \mathrm{M}$ thiourea (Sigma), 4\% (w/v) CHAPS (Sigma) and $30 \mathrm{mM}$ Tris (Sigma) adjusted to $\mathrm{pH} 8.5$ ). After 5 days storage at $+4^{\circ} \mathrm{C}$ or $\mathrm{RT}$, all the samples were preserved at $-20^{\circ} \mathrm{C}$ until needed. As reference, 30 pairs of SGs from each species were collected in two independent replicates and placed on ice in PBS followed by freezing at $-20^{\circ} \mathrm{C}$ [15].

Before testing the protein preservation conditions, the quantity of SG proteins collected was estimated for each sample. As protein degradation could occur in the different conditions tested, 2 pools of 30 pairs of SG for each mosquito species were collected in 2 independent experiments and conserved in reference conditions on ice in PBS. These pooled samples were then used for protein concentration measurements by the Lowry method (DC Protein assay Kit, Bio-Rad) according to the manufacturer's instructions. Protein concentration of An. gambiae s.s. and Ae. Aegypti samples were estimated at an average of $18.1 \pm 2.0 \mu \mathrm{g}$ (mean \pm standard deviation) and $25.0 \pm 2.4 \mu \mathrm{g}$ per tube of $30 \mathrm{SG}$ pairs, respectively.

SGs preserved in each condition were then disrupted by ultrasonication for $5 \mathrm{~min}$ on ice. Each sample was split into two equal quantities and precipitated with cold acetone (Sigma). One protein sample was suspended in bicarbonate buffer $0.1 \mathrm{M}(\mathrm{pH} 9.6)$ at $2 \mu \mathrm{g} /$ $\mathrm{mL}$, suitable for ELISA procedure, and the other was suspended in cell lysis buffer at $2.5 \mu \mathrm{g} / \mu \mathrm{L}$, suitable for biochemical analysis [15]. To avoid several freeze-thaw cycles of the SGE samples and sera, ELISA and immunoblot experiments were run in parallel. Sera from 5 individuals (3 Senegalese and 2 Gabonese) regularly exposed to An. gambiae and Ae. aegypti mosquito bites and sera from 2 non-exposed French individuals who had not travelled abroad for the past 5 years were selected for this study. The protocol was approved by the ethical committee of Marseille (France) and by the Senegal National Ethics Committee (Dakar, Senegal). The informed consent of each participant was obtained at the beginning of the study, after a thorough explanation of its purpose.

ELISA analyses were performed as previously described [21]. Each serum (diluted at 1/50) was tested in duplicate and in control wells without SG extracts. IgG antibody levels are reported as adjusted OD (aOD), calculated for each serum as a mean OD value with SG extracts minus the OD value of the control wells.

A high IgG antibody response against An. gambiae SGs stored in the reference condition was observed by ELISA for exposed individuals (mean aOD \pm standard deviation: $0.73 \pm 0.21$ ), in contrast to non-exposed individuals $(0.15 \pm 0.03)$. Comparable IgG antibody responses were obtained against Ae. aegypti SGs stored in the reference condition $(0.75 \pm 0.29$ and $0.06 \pm 0.01$ for exposed and non-exposed individuals respectively; Figure 1A). These positive sera were considered suitable for the evaluation of the SGs' antigenicity.

The aOD of IgG antibody against SGs of both mosquito species from exposed individuals differed according to storage conditions. For An. gambiae samples, the highest mean aOD from exposed individuals was observed for SGs collected in PBSpi $(0.97 \pm 0.26)$, and to a lesser extent in cell lysis buffer $(0.69 \pm 0.30)$, when maintained for 5 days at $+4^{\circ} \mathrm{C}$. However, for samples collected in these same buffers (i.e., PBSpi or cell lysis buffer) but stored at RT, a decrease in aOD was observed $(0.46 \pm 0.21$ and $0.64 \pm 0.22$ for samples collected in PBSpi and cell lysis buffer respectively; Figure 

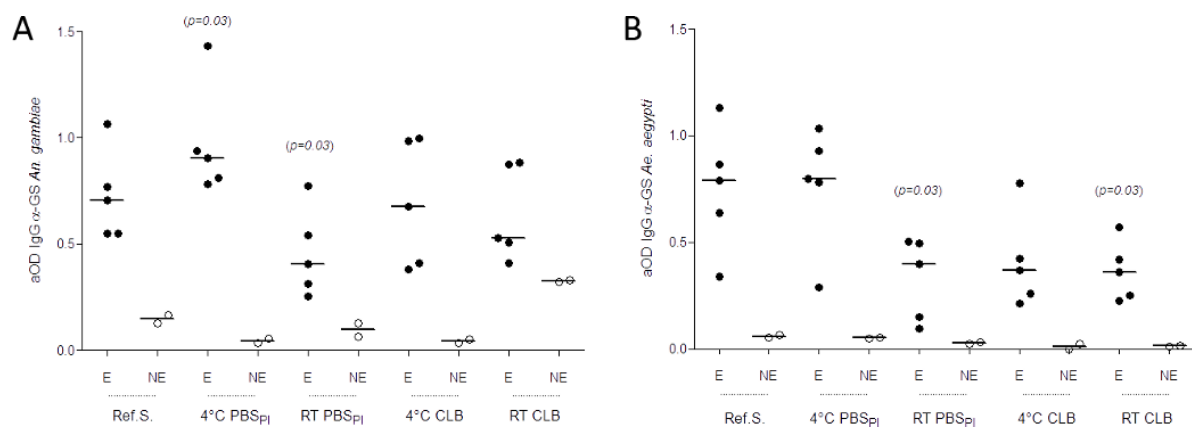

Figure 1 Scatter plot graphs of human IgG responses from exposed (E) and non-exposed (NE) individuals against An. gambiae (A) and Ae. aegypti (B) salivary gland (SG) protein extracts according to different preservation conditions by Enzyme-Linked ImmunoSorbent Assay (ELISA). Antibody responses are represented by aOD: mean OD value of wells with salivary antigen minus mean OD value of wells without salivary antigen. Each point shows the aOD value for a single individual. Horizontal bars show medians. Differences between the reference sample (salivary glands collected on ice and store fresh at $-20^{\circ} \mathrm{C}$ ) and other preservation conditions were tested using Wilcoxon signed-rank test. $p$-values are indicated only when significant differences were observed. Ref. S: reference sample; CBL: cell lysis buffer.

1A). For Ae. aegypti samples, only SGs collected in $\mathrm{PBSpi}$ and stored at $+4^{\circ} \mathrm{C}$ showed an aOD from exposed individuals $(0.77 \pm 0.29)$ similar to that detected under the reference preservation condition $(0.75 \pm 0.29$; Figure 1B). The aOD corresponding to IgG response against An. gambiae and Ae. aegypti SGs from non-exposed individuals was lower than the aOD detected with sera from exposed individuals. The aOD obtained with nonexposed sera against An. gambiae SGs preserved in cell lysis buffers at RT $(0.33 \pm 0.01)$ was unexpectedly higher than that obtained with An. gambiae SGs preserved in the other conditions. For An. gambiae SGs preservation conditions, significant increase and decrease (Wilcoxon signed rank test) of aOD from exposed individual sera were observed between reference sample and PBSpi at $+4^{\circ} \mathrm{C}(p=0.03)$ or PBSpi at RT $(p=0.03)$, respectively. For Ae. aegypti SGs preservation conditions, significant decreases (Wilcoxon signed rank test) of aOD from exposed individual sera were observed between reference sample and PBSpi at RT $(p=0.03)$ or cell lysis buffer at RT $(p=0.03)$ (Figure 1). Collectively, these results indicated that PBSpi at $+4^{\circ} \mathrm{C}$ appeared to be the most efficient medium for preserving $S G$ antigenicity during a 5-day storage period for further analysis by ELISA.

SG preservation was further evaluated by biochemical analysis including comparison of protein profiles by SDS-PAGE and immune response by immunoblots. For each preservation condition, $10 \mu \mathrm{g}$ of SG protein was minimally labeled with CyDye as previously described $[15,22]$ and separated by $12 \%$ SDS-PAGE (BioRad, Hercules, USA). Protein profiles were then analyzed using the ImageQuant ${ }^{\mathrm{TM}} \mathrm{TL}$ software (GE Healthcare, UK), as previously described [23].

For An. gambiae, the diversity of protein bands, compared to the frozen reference, was independent of the preservation conditions used (Figure 2A), but large band intensity variations were observed dependent on the preservation conditions. For the same band, protein abundance between PBSpi RT and cell lysis buffer at $4^{\circ} \mathrm{C}$ could vary up to 11 -fold (Figure $2 \mathrm{~B}$ ). Protein profiles with higher band diversity and intensity were obtained for samples preserved in cell lysis buffer either at $+4^{\circ} \mathrm{C}$ or RT.

To further assess the consequences of sample preservation on the antigenic repertory, gels were transferred onto a nitrocellulose membrane (GE Healthcare) by semidry blotting [24] and further incubated with human pool sera from exposed individuals $(\mathrm{n}=5$, diluted at $1 /$ 100 ) and revealed mouse anti-human Fcg/IgG horseradish peroxidase (HRP) conjugated antibody (1/5 000, Beckman Coulter, USA) using an ECL Plus detection system (GE Healthcare). In accordance with SDS-PAGE analysis, immunoblots indicated that the antigenic repertoire appears better preserved in cell lysis buffer both at $+4^{\circ} \mathrm{C}$ or RT. In fact, numerous antigenic bands were detected only under this last preservation condition (Figure 2C). The intensity and diversity of antigenic profiles were most intense after cell lysis preservation when compared to the other conditions. Similar results were obtained for Ae. aegypti SG samples (Figure 3).

Surprisingly, in the reference condition, the low quality of protein and immune profiles suggested that protein degradation could occur in samples left at $4^{\circ} \mathrm{C}$ during SG collection periods, but the addition of a protease inhibitor cocktail could counteract this deleterious phenomenon. Interestingly, in An. gambiae samples, also the band at $70 \mathrm{kDa}$, reported as a major antigen [25], was detected of in all preservation conditions on the SDS-PAGE, this band was not recognized by the pool sera in the reference and PBSpi at RT conditions. The non-detection of this band could be reasonably 


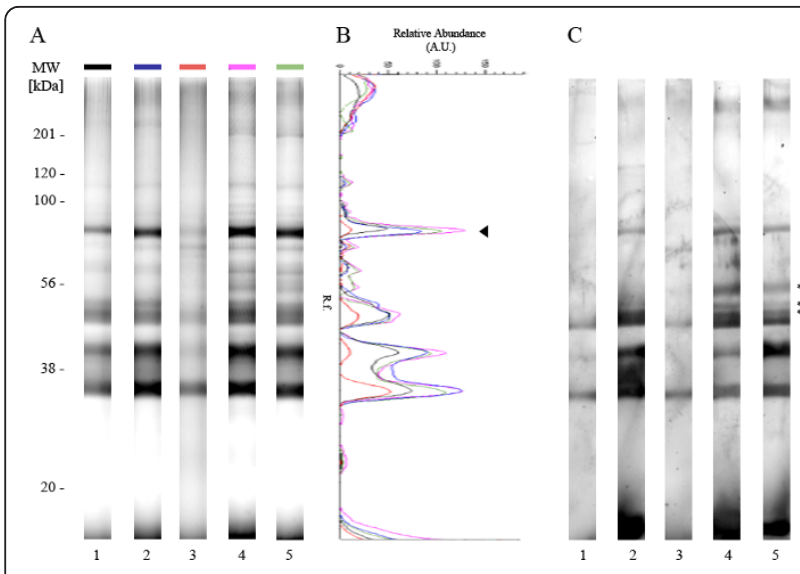

Figure 2 Comparison of An. gambiae salivary gland profiles at the protein and antigenic level according to different preservation conditions. (A) Comparative An. gambiae salivary gland (SG) protein profiles between different preservation conditions. Salivary gland proteins were separated on $12 \%$ SDSPAGE gels. Each protein profile corresponds to a distinct preservation condition. Lane 1: SG dissected on ice and stored at $-20^{\circ} \mathrm{C}$ in PBS (reference); lane 2: SG dissected at RT and stored 5 days at $4^{\circ} \mathrm{C}$ in PBS containing protease inhibitor cocktail (PBSpi); lane 3: SG dissected at RT and stored 5 days at RT in PBSpi; lane 4: SG dissected at RT and stored 5 days at $4^{\circ} \mathrm{C}$ in cell lysis buffer; lane 5: SG dissected at RT and stored 5 days at RT in cell lysis buffer. Standard molecular weights (MW) are indicated at the left side in kilodaltons ( $\mathrm{kDa}$ ). (B) Schematic representations of densitometric protein profiles from the 5 salivary gland preservation conditions. The line color corresponds to the colored box used at the top of each protein profile. The arrow head indicates the band that was used for abundance comparison. A.U.: Arbitrary Unit. R.f.: Relative front of migration. (C) IgG immune profiles against An. gambiae salivary gland proteins using the pooled sera from exposed individuals. The immunoblots were performed by transferring the SDS-PAGE gel shown in (A) onto a nitrocellulose membrane. Antigenic bands detected only in samples preserved in cell lysis buffer are indicated with an asterisk (*).

attributed to an under detection limit of the immunoblot by this pool sera. Effectively, the band at $70 \mathrm{kDa}$ is largely less abundant in the reference and PBSpi at RT conditions compared to three other conditions (PBSpi $+4^{\circ} \mathrm{C}$, cell lysis buffer $+4^{\circ} \mathrm{C}$ or RT) accordingly to their corresponding densitometric protein profiles.

Although cell lysis buffer seems to be the best condition to preserve protein integrity for biochemical analysis at both $+4^{\circ} \mathrm{C}$ and $\mathrm{RT}$, the lower aOD detected by ELISA from samples in these conditions could be attributed to the reagents from the cell lysis buffer (despite the acetone precipitation) interfering with the ELISA reaction, rather than a degradation of protein antigenicity. Effectively, Godfrin and collaborators demonstrate that high concentrations of CHAPS, Urea and Thiourea inhibit antigen binding to microplate surface and could also disturb antigen recognition by the specific antibodies in ELISA [26]. In addition, the

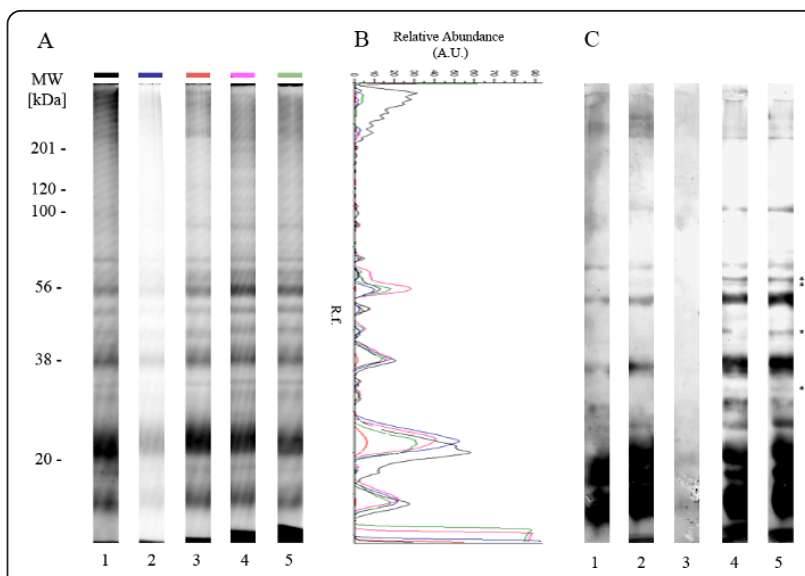

Figure 3 Comparison of Ae. aegypti salivary gland profiles at the protein and antigenic level according to different preservation conditions. (A) Comparative Ae. aegypti salivary gland (SG) protein profiles between different preservation conditions. Salivary gland proteins were separated on 12\% SDS-PAGE gels. Each protein profile corresponds to a distinct preservation condition. Lane 1: SG dissected on ice and stored at $-20^{\circ} \mathrm{C}$ in PBS (reference); lane 2: SG dissected at RT and stored 5 days at $4^{\circ} \mathrm{C}$ in PBS containing a protease inhibitor cocktail (PBSpi); lane 3: SG dissected at RT and stored 5 days at RT in PBSpi; lane 4: SG dissected at RT and stored 5 days at $4^{\circ} \mathrm{C}$ in cell lysis buffer; lane 5: SG dissected at RT and stored 5 days at RT in cell lysis buffer. Standard molecular weights (MW) are indicated at the left side in kilodaltons $(\mathrm{kDa})$. (B) Schematic representations of densitometric protein profiles from the 5 salivary gland preservation conditions. The line color corresponds to the colored box used at the top of each protein profile. A.U.: Arbitrary Unit. R.f.: Relative front of migration. (C) IgG immune profiles against Ae. aegypti salivary gland proteins using the pooled sera from exposed individuals. The immunoblots were performed by transferring the SDS-PAGE gel shown in (A) onto a nitrocellulose membrane. Antigenic bands detected only in samples preserved in cell lysis buffer are indicated with an asterisk (*).

combination of detergent and chaotropic agents in the cell lysis buffer induces protein denaturation leading to the loss of conformational epitopes [27]. The better conservation of these conformational epitopes in PBS is a supplementary argument to explain differences observed between preservation conditions in ELISA. Conversely, in immunoblots, epitopes recognized are mainly sequential due to reduction and denaturation of proteins, which could explain the disparate results obtained between the ELISA reactions and the SDSPAGE or immunoblots.

To summarize, the cell lysis buffer solution seems to prevent protein degradation and preserve antigenicity at $+4^{\circ} \mathrm{C}$ and also at RT. Nevertheless, despite cleaning the samples by acetone treatment, traces of this buffer could disrupt the ELISA experiments. In this specific case, protein preservation under PBSpi appeared to be more efficient to preserve SG antigenic proteins after 5 days of storage at $+4^{\circ} \mathrm{C}$. These convenient storage methods provide an alternative to freezing, which is hard to 
achieve under field conditions, and are expected to be applicable to biological samples in many systems.

\section{Abbreviations}

IgG: Immunoglobulin G; RT: Room temperature; PBS: Phosphate Buffered Saline; PBSpi: Phosphate Buffered Saline supplemented with a protease inhibitor cocktail; EDTA: ethylenediaminetetraacetic acid; ELISA: EnzymeLinked ImmunoSorbent Assay; HRP: Horseradish peroxidase; SDS-PAGE: Sodium dodecyl sulfate polyacrylamide gel electrophoresis.

\section{Acknowledgements}

The authors gratefully acknowledge D. Fontenille and M.N. Lacroix from IRD UR016 for access to mosquitoes bred in the insectary. This study was supported by the French Armed Forces Medical Service and the Délégation Générale pour l'Armement (ARTHROSER project, Grant 10CO401).

\section{Authors' contributions}

Conceived and designed the experiments: FA, AL, FT and RC. Performed the experiments: FA, PA and BS. Analyzed the data: FA, PA, AL and RC. Contributed reagents/materials/analysis tools: $\mathrm{BS}, \mathrm{DI}$ and $\mathrm{BN}$. Wrote the paper: FA, AL and RC. All authors read and approved the final version of the manuscript

\section{Competing interests}

The authors declare that they have no competing interests.

Received: 9 December 2010 Accepted: 8 March 2011 Published: 8 March 2011

\section{References}

1. Tolle MA: Mosquito-borne diseases. Curr Probl Pediatr Adolesc Health Care 2009, 39:97-140.

2. Weaver SC, Reisen WK: Present and future arboviral threats. Antiviral Res 85:328-345.

3. Ribeiro JM: Blood-feeding arthropods: live syringes or invertebrate pharmacologists? Infect Agents Dis 1995, 4:143-152.

4. Arca B, Lombardo F, Valenzuela JG, Francischetti IM, Marinotti O, Coluzzi M, Ribeiro JM: An updated catalogue of salivary gland transcripts in the adult female mosquito, Anopheles gambiae. J Exp Biol 2005, 208:3971-3986.

5. Ribeiro JM, Arca B, Lombardo F, Calvo E, Phan VM, Chandra PK, Wikel SK: An annotated catalogue of salivary gland transcripts in the adult female mosquito, Aedes aegypti. BMC Genomics 2007, 8:6.

6. Andrade BB, Teixeira CR, Barral A, Barral-Netto M: Haematophagous arthropod saliva and host defense system: a tale of tear and blood. An Acad Bras Cienc 2005, 77:665-693.

7. Billingsley PF, Baird J, Mitchell JA, Drakeley C: Immune interactions between mosquitoes and their hosts. Parasite Immunol 2006, 28:143-153.

8. Waitayakul A, Somsri S, Sattabongkot J, Looareesuwan S, Cui L, Udomsangpetch R: Natural human humoral response to salivary gland proteins of Anopheles mosquitoes in Thailand. Acta Trop 2006, 98:66-73.

9. Remoue F, Cisse B, Ba F, Sokhna C, Herve JP, Boulanger D, Simondon F: Evaluation of the antibody response to Anopheles salivary antigens as a potential marker of risk of malaria. Trans R Soc Trop Med Hyg 2006, 100:363-370.

10. Palosuo K, Brummer-Korvenkontio H, Mikkola J, Sahi T, Reunala T: Seasonal increase in human lgE and lgG4 antisaliva antibodies to Aedes mosquito bites. Int Arch Allergy Immunol 1997, 114:367-372.

11. Schneider BS, Higgs S: The enhancement of arbovirus transmission and disease by mosquito saliva is associated with modulation of the host immune response. Trans R Soc Trop Med Hyg 2008, 102:400-408.

12. Titus RG, Bishop JV, Mejia JS: The immunomodulatory factors of arthropod saliva and the potential for these factors to serve as vaccine targets to prevent pathogen transmission. Parasite Immunol 2006, 28:131-141.

13. Valenzuela JG, Francischetti IM, Pham VM, Garfield MK, Ribeiro JM: Exploring the salivary gland transcriptome and proteome of the Anopheles stephensi mosquito. Insect Biochem Mol Biol 2003, 33:717-732.
14. Choumet V, Carmi-Leroy A, Laurent C, Lenormand P, Rousselle JC, Namane A, Roth C, Brey PT: The salivary glands and saliva of Anopheles gambiae as an essential step in the Plasmodium life cycle: a global proteomic study. Proteomics 2007, 7:3384-3394.

15. Almeras L, Fontaine A, Belghazi M, Bourdon S, Boucomont-Chapeaublanc E, Orlandi-Pradines E, Baragatti M, Corre-Catelin N, Reiter P, Pradines B, Fusai T, Rogier C: Salivary gland protein repertoire from Aedes aegypti mosquitoes. Vector Borne Zoonotic Dis 2010, 10:391-402.

16. Ribeiro JM, Arca B: From sialomes to the sialoverse: an insight into the salivary potion of blood feeding insects. Adv Insect Physiol 2009, 37:59-118.

17. Arca B, Lombardo F, de Lara Capurro M, della Torre A, Dimopoulos G, James AA, Coluzzi M: Trapping CDNAs encoding secreted proteins from the salivary glands of the malaria vector Anopheles gambiae. Proc Natl Acad Sci USA 1999, 96:1516-1521.

18. Gerberg J: Manual for mosquito rearing and experimental techniques. American Mosquito Control Association 1970, Bulletin No. 5.

19. Mahama T, Desiree EJ, Pierre C, Fabrice C: Effectiveness of permanet in Cote d'Ivoire rural areas and residual activity on a knockdown-resistant strain of Anopheles gambiae. J Med Entomol 2007, 44:498-502.

20. Marcombe S, Darriet F, Agnew P, Etienne M, Yp-Tcha MM, Yebakima A, Corbel V: Field efficacy of new larvicide products for control of multiresistant Aedes aegypti populations in Martinique (French West Indies) Am J Trop Med Hyg 2011, 84:118-126.

21. Orlandi-Pradines $E$, Almeras L, Denis de Senneville L, Barbe S, Remoue F, Villard C, Cornelie S, Penhoat K, Pascual A, Bourgouin C, Fontenille D, Bonnet J, Corre-Catelin N, Reiter P, Pagés F, Laffite D, Boulanger D, Simondon F, Pradines B, Fusaï T, Rogier C: Antibody response against saliva antigens of Anopheles gambiae and Aedes aegypti in travellers in tropical Africa. Microbes Infect 2007, 9:1454-1462.

22. Pastorino B, Boucomont-Chapeaublanc E, Peyrefitte CN, Belghazi M, Fusai T, Rogier C, Tolou HJ, Almeras L: Identification of cellular proteome modifications in response to West Nile virus infection. Mol Cell Proteomics 2009, 8:1623-1637.

23. Almeras L, Orlandi-Pradines E, Fontaine A, Villard C, Boucomont E, de Senneville LD, Baragatti M, Pascual A, Pradines B, Corre-Catelin N, Pages F, Reiter $\mathrm{P}$, Rogier C, Fusai T: Sialome individuality between Aedes aegypti colonies. Vector Borne Zoonotic Dis 2009, 9:531-541.

24. Towbin H, Staehelin T, Gordon J: Electrophoretic transfer of proteins from polyacrylamide gels to nitrocellulose sheets: procedure and some applications. 1979. Biotechnology 1992, 24:145-149.

25. Cornelie S, Remoue F, Doucoure S, Ndiaye T, Sauvage FX, Boulanger D, Simondon F: An insight into immunogenic salivary proteins of Anopheles gambiae in African children. Malar J 2007, 6:75.

26. Godfrin D, Senechal H, Sutra JP, Busnel JM, Desvaux FX, Peltre G: A modified enzyme-linked immunosorbent assay adapted for immunodetection of low amounts of water-insoluble proteins. J Immunol Methods 2007, 326:83-92

27. Rabilloud T, Gianazza E, Catto N, Righetti PG: Amidosulfobetaines, a family of detergents with improved solubilization properties: application for isoelectric focusing under denaturing conditions. Anal Biochem 1990, 185:94-102.

doi:10.1186/1756-3305-4-33

Cite this article as: Fontaine et al: Mosquito salivary gland protein preservation in the field for immunological and biochemical analysis. Parasites \& Vectors 2011 4:33. 